\title{
Politische Heiraten im Alten Orient
}

\author{
Von \\ WOLFGANG RƠLLIG \\ Tübingen
}

Als Alexander der Große 328 v. Chr. den „Soghdischen Felsen“ des Oxyartes erobert hatte, befand sich unter den Gefangenen auch Roxane, die Tochter des Fürsten, die er wenig später nach makedonischem Ritus heiratete, weil er ,glaubte, daß es nach den gegebenen Verbältnissen nicht unpassend sei" ${ }^{\text {"1 }}$, und die ihm schließlich seinen Sohn Alexander IV. posthum gebar. Nach der Rückkehr aus Indien setzte er 324 gar die berühmte Massenhochzeit von Susa in Szene, „bei der etwa 80 vornebme Makedonen je nach ibrer Stellung im Heer mit persischen Prinzessinnen bzw. Töchtern der persischen Aristokratie vermählt wurden ${ }^{2}$ und 10000 Soldaten ihre asiatischen Konkubinen heirateten. Er selbst heiratete bei dieser, nun nach persischem Ritus vorgenommenen Zeremonie, Barsine (oder Stateira), eine Tochter seines einstmals größten Widersachers, Dareios III. Es ist viel über diese Hochzeiten geschrieben, die ihnen zugrundeliegende Tendenz ist verschieden interpretiert worden: Rassenmischung zwischen den Herrenvölkern der Griechen und der Perser, Verschmelzungspolitik Alexanders zwischen Orient und Okzident, Versuch der Versöhnung aller im Alexanderreich zusammengefaßten Völker, ja der Gedanke einer allgemeinen Weltverbrüderung wurde dem König unterstellt. Wie dem auch sei, eines ist deutlich: Beide Hochzeiten, diejenige mit Roxane und die Massenhochzeit in Susa, sind politischer Natur gewesen, „es war den gegebenen Verbältnissen nach nicht unpassend“, wie Plutarch schon mit Recht interpretierte. Es läßt sich aber auch zeigen, daß Alexander sich hier in der Nachfolge einer Politik befindet, die im Vorderen Orient eine lange Tradition hat. Davon soll im folgenden die Rede sein.

Wir dürfen unterstellen, daß Hochzeiten zwischen Herrscherhäusern, zumal im Alten Orient, keine „Liebesheiraten" waren, sondern mit politischer Absicht erfolgten. Verschiedene Absichten konnten dabei bestimmend sein: Waren beide Partner politisch gleich stark, so konnte das Knüpfen familiärer Bande die gegenseitige Beziehung neutral halten. War einer der Partner stärker als der andere, so konnte er eine Tochter vergeben, um den schwächeren an sich zu binden; der schwächere Partner konnte eine Königstochter anbieten, um sich den Schutz des Stärkeren zu sichern. Auch der Prestigegewinn, der mit der familiären Bindung an ein oder mehrere Fürstenhäuser verbunden war, soll nicht unterschätzt werden. Schließlich konnten politische Heiraten dazu benutzt werden, um Völkerschaften oder Stämme zu integrieren, die neu in das Reichsgebiet eingegliedert wurden oder zu solch mächtigen Faktoren im politischen Kräftespiel geworden waren, daß sie nicht länger ignoriert werden konnten. Alle diese Motivationen gibt es bereits im Alten Orient, und es ist reizvoll, einmal die verschiedenen Verbindungen nachzuzeichnen, die die Überlieferung uns nennt.

Einige Einschränkungen sind zunächst zu machen: Wir kennen eine ganze Reihe von dynastischen Heiraten, sicher aber nur einen kleinen Prozentsatz derer, die tatsächlich

1 Plutarch, Alexander 47. Vgl. W. W. Tarn, Alexander der Große (Darmstadt 1968) S. 79, 313.

2 H. E. Stier, Welteroberung und Weltfriede im Wirken Alexanders des Großen, in: RheinischWestfälische Akademie der Wissenschaften, Vorträge G 187 (1973) S. 40; dort auch zum Folgenden. Vgl. ferner W. W. Tarn, op. cit. (Anm. 1) S. 114; J. Seibert, Alexander der Große, in: Erträge der Forschung 10 (1972) S. 186 ff. 
durchgeführt worden sind. Die Nachrichten über familiäre Ereignisse, auch wenn sie in die Politik hinüberspielten, sind in der altorientalischen Literatur so selten, daß oft nur zufällige Erwähnungen uns auf ihre Spur bringen. Aber auch wenn - wie selten - ein Jahresname nach dem Vollzug einer politischen Heirat gewählt wurde, ist über die Motivation, die dieser Heirat zugrunde lag, nichts zu erfahren. Wir können allerdings aus unserer Kenntnis der allgemeinen politischen Situation heraus zuweilen diese Motivation im groben erschließen. Detaillierte Schilderungen solcher Heiraten gibt es nicht, einige ausführlichere Korrespondenzen der Amarna-Zeit lassen immerhin über die Motivation der Herrscher dieser Zeit kaum Unklarheiten. Es mag auch sein, daß ich in der fast unübersehbaren Literatur den einen oder anderen wichtigen Beleg übersah und deshalb ein Aspekt fehlt. Ausdrücklich ausgeklammert ist alles, was mit der „kultischen Hochzeit“ zusammenhängt, auch wenn diese oft genug auch „politisch" gewesen sein dürfte.

Ein erstes Beispiel soll zeigen, wie beide Bereiche, der politische und der religiöse, auch nach der Vorstellung der Sumerer ineinander flossen; wie "politische "Vorgänge im Vollzug der Eheschließung auch ihre religiöse Darstellung und Rechtfertigung fanden.

Geschichte beginnt im Mythos. Wie könnte es deshalb anders sein, als daß auch politische Heirat sich hier manifestiert. Schlecht überliefert, aber doch hinreichend verständlich ist der Martu-Mythos ${ }^{3}:$ Martu, Gott der in der Steppe herumstreifenden Nomaden, offenbar in der Stadt Ninab ansässig und unverheiratet, macht sich auf die Suche nach einer Frau. Anläßlich eines großen Festes in Ninab kommt auch der Gott Numušda von Kazallu mit seiner Familie, er ist von bestimmten Taten des Martu beeindruckt und möchte ihm ein Angebot machen. Martu wählt sich eine Tochter des Numušda zur Frau, die - obwohl von ihren Gespielinnen mit Hinweis auf die rohe Lebensart der Nomaden vor ihm gewarnt auch in die Ehe einwilligt. Die Ätiologie für den Mythos ist einsichtig: Martu wird durch die Ehe mit einer Tochter des gut sumerischen Numušda in dessen Familie und damit in das Pantheon aufgenommen. Politisch gewendet heißt das: Aufnahme der Nomaden in die Zivilisation.

Dieses Beispiel mag genügen, um zu zeigen: Die familiäre Bindung, die mit einer Hochzeit eingegangen wird, braucht nicht nur den privatrechtlichen Bereich zu berühren, sondern kann darüber hinaus wirken - und das wurde auch von den Sumerern bereits so verstanden.

Politische Heiraten in großem Umfang, nämlich zur Festigung einer Dynastie, kann ich erst bei Gudea von Lagaš (um 2100 v. Chr.) nachweisen, hier allerdings schon in erstaunlichem $\mathrm{Maße}^{4}$. Nach der Rekonstruktion A. Falkensteins ${ }^{5}$ wird Gudea deshalb zum Stadtfürsten von Lagaš, weil er die Ninalla, Tochter des Urbaba, Stadtfürsten von Lagaš, zur Frau nahm. Er heiratet also in die Dynastie ein. Gleichfalls eine Tochter des Urbaba hei-

${ }^{3}$ Publikation: E. Chiera, Sumerian Epics and Myths (1934) Nr. 58; vgl. ders. Sumerian Religious Texts (1924) S. 14-23; S. N. Kramer, Sumerian Mythology (2. Aufl. 1961) S. 98 ff.

${ }^{4}$ Ein erstes Zeugnis dynastischer Heirat liegt aus dem Nachbarstaat von Lagaš, aus Umma vor. Hier heiratete der König Gišškakidu (um 2390 v. Chr.) die Barairnun, Tochter des Urlumma (nach F. Thureau-Dangin, in: Rev. d'Assyriologie 34 [1937] S. $178=$ E. Sollberger/J.-R. Kupper, Inscriptions royales sumériennes et akkadiennes [1971] ID 5). Da dieser Urlumma aber sein Großonkel und ebenfalls regierender Fürst war, diente diese Heirat mit einer Nichte zweiten Grades wohl der familiären Verklammerung und damit der Festigung der Dynastie.

${ }^{5}$ In: Die Inschriften Gudeas von Lagaš I = Analecta Orientalia 30 (1966) S. 1 ff. Das dort ermittelte Stemma der genealogischen Verknüpfungen sei hier zur Verdeutlichung angeschlossen. In Klammern die - vermutliche - Folge der Dynasten. Einige Fragen der Genealogie (und Chronolo- 
ratete $U_{r-G A R}$, ein späterer Stadtfürst am gleichen Ort, der sogar noch mit Nin-KA-gina, einer Tochter des Bruders des Urbaba, in zweiter Ehe verbunden war. Schließlich nahm Nammabani, Nachfolger des $U r-G A R$, die Ninbedu zur Frau, die gleichfalls eine Tochter Urbabas war, vielleicht aber auch noch seine Nichte, wenn sein Vater Lugirizal tatsächlich der ältere Bruder des Urbaba war. Wir haben hier also den etwas kuriosen Fall vor uns, $\mathrm{daß}$ der 4., der 7. und der 8. Herrscher einer Dynastie mit Töchtern des 3. Herrschers verheiratet, also alle miteinander verschwägert waren. Es ist klar, daß eine solche Heiratspolitik außerordentlich systemstabilisierend wirkte, auch wenn mit Sohn und Enkel des Gudea die Herrscherfolge der Schwager durchbrochen ist.

Wir dürfen aber annehmen, daß diese starke Betonung der familiären Bindung für politische Zwecke nicht auf die friedliche Dynastie von Lagaš beschränkt blieb. Wenig später betreibt die 3. Dynastie von Ur offenbar eine regelrechte Heiratspolitik, nun aber nicht mehr nach innen, zur Stützung der eigenen Dynastienfolge, vielmehr nach außen. $\check{S}$ ulgi vermählt in seinem 18 . Regierungsjahr - selbst die Datenformel wurde danach gewählt - seine Tochter mit dem Fürsten von Marhaši, einem Land, das an Elam angrenzte ${ }^{6}$, vergibt dann in seinem 31. Jahr auch noch eine Tochter an den ensi von Anšan ${ }^{7}$, was ihn nicht hinderte, im 34. Jahre "die Zerstörung von Anšan" zu melden, die entweder nur wenig wirksam war oder mit einer Rückführung der Königstochter einherging. Der jüngere Sohn $\breve{S} u l g i s, \check{S} \bar{u}$-Sîn, sendet in seinem zweiten Jahr eine seiner Töchter mit einem Gesandten des Fürsten von Anšan mit reichem Troß nach dem Osten ${ }^{8}$, und auch der letzte König dieser Dynastie, der unglückliche $I b b i-S i n$, führt diese Tradition fort: Seine Tochter Tukin-hatti-migrǐ̌sa wird vom ensi von Zabšali zur Frau genommen ${ }^{9}$. Selbst $I^{2} b \bar{i}$-Erra, der Mann aus Mari, Gegenspieler des Ibbi-Sîn, behält diese Politik bei. Wir kennen die Mitgift seiner Tochter $L \bar{\imath} b \bar{u} r-n \bar{\imath} r u m^{10}$, die dem $I^{\nu}{ }^{v} d u m-k \bar{e} n$ zur Ehe gegeben wurde, einem Sohn des Hubasimti, der wiederum ein Sohn des Hutrantemti von Elam war, der mit seinem Überfall auf Ur die alte Dynastie beseitigte. Wenn außerdem bekannt ist, daß I ${ }^{v} b \bar{i}-$

gie) sind noch offen, vgl. abweichend z. B. H. Sauren, Zeitschrift d. Deutschen Morgenländischen Gesellschaft Suppl. 1 (1969) S. 115 ff.

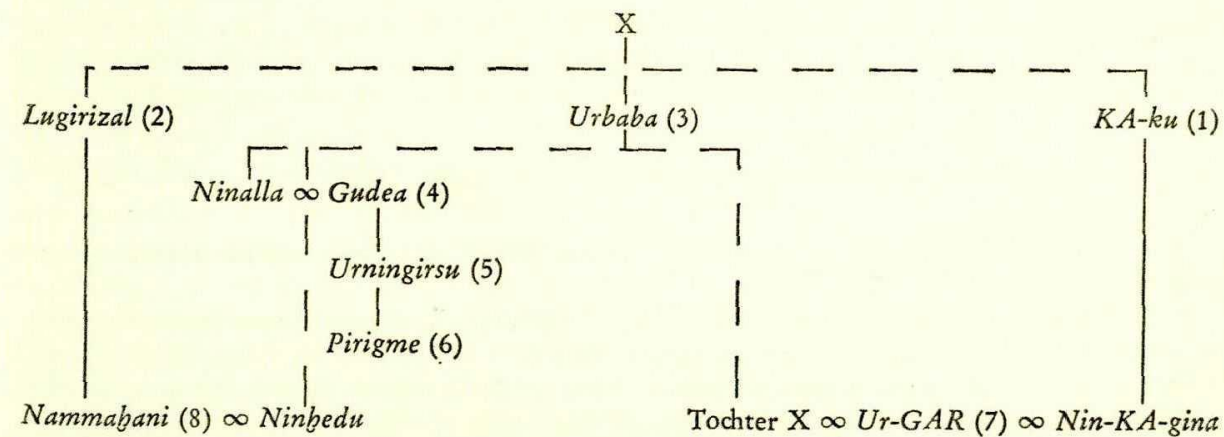

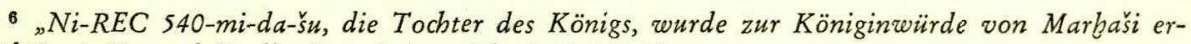
höht": A. Ungnad, Reallexikon d. Assyriologie 2. 141:35.

7 Ebd. 141: 49, vgl. N. Schneider, in: Analecta Orientalia 13 (1936) $15: 28$.

${ }^{8} \mathrm{Ch}$. Virolleaud, in: Zeitschr. f. Assyriologie 19 (1905) S. 384.

9 Jahresdatum des 5. Regierungsjahres Ibbi-Sins, A. Ungnad, op. cit. (Anm. 6) 146:111, s. Th. Jacobsen, in: Journal of Cuneiform Stud. 7 (1953) $37^{9}$.

${ }^{10}$ Die Wirtschaftsurkunde mit Angabe der Mitgift ist publiziert von V. E. Crawford, Bab. Inscr. Nies Bd. 9 (1954) Nr. 438. Zur elamischen Geschichte dieser Zeit s. W. Hinz, Das Reich Elam (1964)

S. 69 ff.; ders., Cambridge Ancient History, Vol. 1/2, 3. Aufl. 1971, S. 654 ff. 
Erra im gleichen Jahr „Elam mit der Waffe schlug", so ist deutlich, daß hier eine Politik getrieben wird, die alle Mittel benutzt, Krieg und Verschwägerung, um das politische Gleichgewicht zwischen Sumer und Elam aufrechtzuerhalten. Fragen wir nach der Qualität dieser in der Ur-III-Zeit geschlossenen dynastischen Ehen, so sind sie als Versöhnungsoder Beschwichtigungsversuche zu werten. Dauerhafte Verbindungen zwischen Ur und den Nachbarn im Osten scheinen nicht beabsichtigt, eine echte verwandtschaftliche Beziehung nicht aufgenommen worden zu sein.

Das ist offenbar anders in der folgenden Zeit babylonischer Kleinstaaterei. Sumula'el von Babylon, der zweite Herrscher der 1. Dynastie (1816-1780), gab seine Tochter Šallurtum dem Fürsten Sîn-kāšsid von Uruk zur Frau ${ }^{11}$. Das war kein großes historisches Ereignis, denn hier verbanden sich zwei Dynastien, die damals noch völlig bedeutungslos waren, von denen auch nur die von Babylon einmal eine Rolle spielen sollte. Dennoch bedeutet diese Heirat den beteiligten Familien in diesem Stadium offenbar sehr viel. Wir kennen einen ausführlichen Brief des Fürsten Anam von Uruk an Sîn-muballit von Babylon, in dem verschiedene Versehen und Versäumnisse beklagt werden. Dies geschieht aber immer mit dem Hinweis, daß "Uruk und Babylon e in Haus sind", d. h. zusammengehören und keine gegenseitigen Beschuldigungen oder Benachteiligungen kennen sollten ${ }^{12}$. Hier herrscht also ein durchaus familiärer Ton, der wahrscheinlich in der nomadischen Tradition beider Königsfamilien seinen Ursprung hat.

Eine weitere dynastische Ehe, die in ihrer Bedeutung sicherlich nur ganz gering einzuschätzen ist, wurde in Rāpiqum geschlossen, einem etwas oberhalb von Babylon am Euphrat gelegenen Orte. Aus Iščāli (alt: Nērebtum) und aus Sippar stammen je eine Urkunde mit dem Datum: „Jahr: Er nabm die Tochter des Königs zur Ehe nach Räpiqum"13, wobei wir weder den Brautvater noch den Bräutigam kennen. Als Brautvater wurde oft Daduša von Ešnunna vermutet. Aus Tell Harmal, altem Šaduppûm im Einzugsbereich von Bagdad, ist inzwischen eine Datenformel bekannt geworden, die besagt: „[Sîn-a]büsu gab seine Tochter nach Räpiqu", die also ebenfalls eine dynastische Heirat zwischen dem Fürsten $\operatorname{Sin}-a b \bar{u}_{s}^{v} u$ von Šaduppûm und Rāpiqum bezeugt. Es ist wahrscheinlich, daß beide Daten sich auf dasselbe Ereignis beziehen ${ }^{14}$, das in eine Zeit fiel, die einen Machtverfall von Ešnunna sah, so daß Sinn-abǚsu selbst über Nērebtum regierte. Das war kurz vor Daduša, d. h. ca. 1770 v. Chr. Mehr ist von dieser Heirat, die offenbar die Grenze gegen Babylon sichern sollte, nicht bekannt. Auch eine wesentlich frühere dynastische Heirat, die der Tochter Bilalamas von Ešnunna (um 1915) namens ME-kubi (oder Simat-kubi) mit Tanruburatir, dem Stadtfürsten von Susa, ist lediglich zu registrieren ${ }^{15}$. Daß der Staat

${ }^{11}$ Abzuleiten aus der Abrollung einer Rollsiegelinschrift, s. A. Falkenstein, in: Baghdader Mitteilungen 2 (1963) S. 6 f.

12 "Seit die Könige von Uruk und Babylon e in, Haus' sind - abgesehen vom jetzigen Zeitpunkt, in dem ich und du uns erzürnt haben -, und nach dem, was ich von meinem Vater und Großvater, die ich gekannt babe, aus mündlicher Mitteilung immer wieder gebört babe, seit Sînkāsid, seit ich es weiß, bis jetzt, sind die Truppen der (Stämme) Amnän-Jabrur zweimal, dreimal zur Hilfe für dieses ,Haus' bierber gekommen", A. Falkenstein, ebd. 62, Z. 25-32, s. S. 25 f.

${ }^{13}$ H. F. Lutz, University of California Publ., in: Semitic Philology 10 (1931) S. 134, Nr. 61,10f. und V. Scheil, Une saison de fouilles à Sippar (1902) S. 127 Nr. 237.

${ }_{14}$ Die Tell Harmal-Urkunde wurde publiziert von J. van Dijk, Texts in the Iraq Museum Vol. 3 (1966) Nr. 30, s. F. Reschid, Archiv des Nūršamaš und andere Darlehensurkunden aus der altbabylon. Zeit (Diss. Heidelberg 1965) S. 59 und Diskussion auf S. 11. - Das gleiche Archiv (F. Reschid, op. cir. Nr. 31 Datum) bezeugt auch die Heirat einer Tochter des Sîn-abüšsu von Šaduppûm mit dem Fürsten von Mankisum.

15 Ziegelinschrift, s. zuletzt E. Sollberger/J.-R. Kupper, op. cit. (Anm. 4) IVO 2 a; vgl. auch D. O. Edzard, Die, zweite Zwischenzeit' Babyloniens (1957) S. 72 mit Anm. 347 f. 
von Wārum, d. h. Ešnunna, enge Beziehungen zu Elam hatte, wissen wir aus vielen Texten; wie sie sich auswirkten, ist jedoch nicht bekannt.

$*$

Das durch das Mari-Archiv so unvergleichlich gut bekannte Zeitalter des Aufstiegs der Macht Babylons ist nicht nur durch seine stark verzahnte Diplomatie bemerkenswert, sondern hat auch zu zahlreichen politischen Heiraten geführt. Zunächst war Šamš̌̀-Adad von Assyrien die beherrschende Figur. Über seine Frau wissen wir nichts, wohl aber über seine Söhne. Iśme-Dagān, der energische Unterkönig im Osten des Reiches, hat an der Ostgrenze im Zagros-Vorland mit den Turukkäern Frieden geschlossen und als sichtbares Zeichen dafür die Tochter des Zazija als Frau für seinen Sohn Müt-Asqur genommen, selbst den Brautpreis in Gold und Silber entrichtet ${ }^{16}$. Wir wissen durch die dänischen Ausgrabungen in Shemshāra (alt: Šūsarrā) ${ }^{17}$, daß die Turukkäer ein Bergstamm waren, der im heutigen Kurdengebiet wohnte und dem Unterkönig schwer zu schaffen machte. Hier diente die Ehe mit dem Thronprätendenten von Assur zur festen Besiegelung des Friedensschlusses, und die Absicht tut sich darin kund, das gegenseitige Verhältnis durch die Heirat enger zu gestalten. Dadurch, daß ein regelrechter Heiratskontrakt abgeschlossen wird - der Austausch des Brautpreises läßt darauf schließen -, wird weiter deutlich, daß der Turukkäer durchaus selbständig blieb, nicht als Vasall, sondern als freundschaftlich verbundener Nachbar seine Tochter gab. Daß dieses Ereignis, das sich ganz im Osten abspielte, als politisches begriffen wurde, ist leicht daraus ersichtlich, daß man es für wert hielt, es dem König Zimrī-Lim von Mari anzuzeigen.

Jasmab-Adad war der zweite Sohn $\check{S}_{a} m^{\llcorner} \grave{s} \overline{-}-A d a d s$ und Vizekönig in Mari vor der Rückeroberung durch Zimri-Lim. Sein Herrschaftsbereich wies nach Westen, und es ist deshalb nicht sehr verwunderlich, daß er dort schon in jungen Jahren und wahrscheinlich kurz nach der Einsetzung als König eine dynastische Ehe eingehen mußte, nämlich mit der Tochter des $I^{v} b \bar{z}-A d a d$ von Qatna ${ }^{18}$. Hier ist nun die politische Absicht unverkennbar und läßt sich aus den Briefen ablesen: Mari und Qatna konnten gemeinsam einen Riegel vor Expansionsbestrebungen von Jamhad/Aleppo schieben, das unter Sumunpub mehrfach mit dem südwestlich angrenzenden Qatna in Streit geraten war. Mit Hilfe des starken Nachbarn Šam̌̌ī-Adad bzw. Jasmab-Adad möchte $I^{\check{s}} b \bar{b}$-Adad Widerpart bieten können. Er fordert seinen Schwiegersohn, nachdem es nach zähen Verhandlungen um den Brautpreis zur Hochzeit gekommen war, mehrfach auf, ihm mit Truppen zu Hilfe zu kommen. Es ist nicht verwunderlich, daß unter diesen Umständen die Ehe nicht besonders glücklich war, Jasmab-Adad sich mit dem Gedanken trug, seine Frau zu verstoßen. Das wird ihm allerdings von seinem Vater mit Hinweis auf die möglichen politischen Folgen verwehrt. So schiebt er sie nur in den "Palast der Palmen" in Mari ab"

Völlig anders war offenbar das Verhältnis des Zimrī-Lim zu seiner Gattin Šibtu. Auch dies war eine politische Heirat. Bekanntlich hatte Zimri-Lim noch in jungen Jahren am Hofe von Jamhad/Aleppo Schutz gefunden, von hier aus seine Rückeroberung von Mari unternommen. $\mathrm{Zu}$ einem nicht genauer bestimmten Zeitpunkt, wahrscheinlich aber kurz

16 Archives Royales de Mari 2 (1950) Nr. 30.

17 J. Laessøe, The Shemshāra Tablets. A Preliminary Report (1959). - Auch von einem der Fürsten von Šǔšarrā namens Kuwari wird eine politische Ehe eingeleitet: Er gibt seine Tochter dem Sohne eines von $\mathrm{ihm}$ abhängigen Kleinfürsten, „damit die Verschwägerung zwischen uns nicht abreißt" , s. J. Laessøe, op. cit. S. 61 ff., Z. $30 \mathrm{ff}$.

18 Archives Royales de Mari 1 (1950) Nr. 24, 46, 77, vgl. H. Klengel, Geschichte Syriens im 2. Jahrtausend 2 (1969) S. 118 f.

19 G. Dossin, in: Académie de Belgique. Bulletin de la classe de lettres, Bruxelles 1954, S. 421 f. 
nach der Thronbesteigung, heiratete er dann eine Tochter des Jarīm-Lim, seines „Vaters“20, so daß die engen Beziehungen der beiden Herrscherhäuser noch mehr gefestigt wurden. Politisch war dies eine sehr sinnvolle Tat, denn Mari und Jamhad zusammen sperrten den Weg von Mesopotamien nach Syrien, beherrschten die Handelswege und die Wanderwege der Nomaden und waren damit in einer Schlüsselposition. Die Korrespondenz der Frauen aus dem Mari-Palast, die allein 45 Briefe von oder an Šibtu enthält, läßt uns Einblick nehmen in die rege Anteilnahme der Šibtu an Politik und Zeitgeschehen, vor allem aber dem Wohlergehen ihres Gatten. So wird es recht verständlich, wenn dieser ihr einmal schreibt: „Weil du mir schriebst, ich solle eiligst beimkommen, nun, rasch will ich zum Istaropfer beimkommen ${ }^{21}$."

Bald sind schon Töchter vorhanden, mit denen Zimri-Lim seinerseits Heiratspolitik machen kann. So wird eine von ihnen namens Kirum „auf den Königinnenthron" gesetzt ${ }^{22}$, allerdings ohne Angabe darüber, wo und bei wem das geschah. Eine andere Tochter des Königs, Tizpatum, bittet ihren Vater um Hilfe mit der Begründung: „Um meinetwillen befassen sie sich mit dem Manne und meinen: Wie, ist er mit einer Tochter ZimriLims verheiratet und gehorcht nun Zimri-Lim?!23" Hier ist nun klar ausgesprochen, was nur zu oft das Motiv der Heiratspolitik war: Gehorsam zu erhalten oder zu erzeugen. Die Anwesenheit der fremden Königstochter am Hofe wird allein schon Anlaß genug gewesen sein, ständig an die Verbindung mit dem verwandten Herrscherhaus erinnert zu werden. $\mathrm{Daß}$ dies auch lästig sein konnte, haben wir schon gesehen (s. auch unten S. 18). Ein System dynastischer Heiraten konnte aber den Frieden besser sichern, als Verträge das je tun konnten. Deshalb haben auch kleine Staaten immer mehr zu diesem einfachen diplomatischen Mittel gegriffen. Ammitakum von Alalab, der etwa um 1740 regierte, war mit der Tochter eines Regenten von Apišal im Osttigrisland verheiratet ${ }^{24}$, hatte allerdings daneben noch eine Tochter seines Veziers Nawaratal zur Ehe. Ein Jahresdatum meldet aber auch, daß „Ammitakum, der König, ... die Tocbter des Mannes von Ebla für seinen Sobn erwäblte ${ }^{\text {25, also die Verbindung zu einem Nachbarstaat }}{ }^{26}$ auf diesem Wege herstellte oder festigte.

Die Uberlieferung zur Geschichte des Alten Reiches der Hethiter ist nicht besonders reich. Dennoch sind uns Fakten bekannt, die deutlich machen, daß auch hier eine Ehe sehr wesentlich werden konnte, wenn es um das Überleben im politischen Kräftespiel ging. Muršili I., der 1530 Babylon eroberte, hatte eine Schwester namens Harapšili, die mit dem Mundschenken Hantili verheiratet war ${ }^{27}$. Als nun Muršili nach seiner Rückkehr aus Babylon ermordet wurde, folgte ihm Hantili wenn nicht auf dem Thron - er trägt nie den

${ }^{20}$ Diese Heirat ist aus einem Siegel erschließbar, s. H. Klengel, op. cit. (Anm. 18) 1 (1965) S.120; P. Artzi-A. Malamat, in: Orientalia 40 (1971) S. 75-89.

21 Archives Royales de Mari 10 (1967) Nr. 120, Z. 17-20.

22 Ebd. Nr. 34 Z. 8'ff.

23 Ebd. Nr. 98, 13-16.

24 D. J. Wiseman, The Alalakh Tablets (1953) Nr. 409: Liste von Schenkungen(?) anläßlich der Hochzeit.

25 Wiseman, ebd. Nr. 35.

${ }^{26}$ Ebla ist wohl nicht identisch mit dem Tell Mardīh, etwa auf halbem Wege zwischen Aleppo und Hama, vgl. M. C. Astour, Ugarit-Forschungen 3 (1971) S. 9 ff., anders G. Pettinato, in: Annales Archéologiques Arabes Syriennes (1970) S. $19 \mathrm{ff}$.

${ }^{27}$ Telipinu-Erlaß $\ 10$. Zur Diskussion, ob Harapšili die Schwester oder die Gattin (so der Text, doch sind die Keilschriftzeichen für "Schwester" und „Gattin " nur geringfügig verschieden) des Muršili war, s. zuletzt K. Riemschneider, in: Beiträge zur sozialen Struktur des Alten Vorderasien (1971) S. $85^{33}$. 
Königstitel -, so doch in der Machtausübung nach, sicherlich deshalb unbestritten, weil er mit dem Ermordeten verschwägert war. In der Folgezeit, die durch politische Morde und innere Unruhen gekennzeichnet ist, kann sich zweimal ein Thronprätendent dadurch halten, daß er mit Töchtern aus dem eben regierenden Geschlecht verheiratet ist: Zidanta hat eine Tochter Hantilis zur Frau ${ }^{28}$, Telipinu, der mit seinem berühmten „Erlaß" eine Neuordnung im Inneren versucht, ist mit Isstaparija, der Tochter seines Vorgängers Huzzija verheiratet ${ }^{29}$. Es spielt hier allerdings mit hinein, daß die Hethiter offenbar ein matrilineares Thronfolgeprinzip kannten, in das hier jeweils auch Schwägerinnen eingesetzt werden konnten ${ }^{30}$.

Hohe Zeit der politischen Heiraten war dann wieder die Amarnazeit, und hiervon zu berichten ist fast überflüssig. Schon die Amarna-Korrespondenz enthält in ihren ersten Schreiben ${ }^{31}$ zahlreiche Hinweise auf eheliche Verbindungen zwischen den bedeutenden Herrscherhäusern der Zeit, neues Material ist inzwischen hinzugetreten. Kurigalzu I. (ca. 1415-1390) gab eine seiner Töchter an Amenophis III. ${ }^{32}$, der sie in seinen Harem schickte. Kadašman-Enlil nimmt dann diese Beziehung auf, bietet selbst eine seiner Töchter an, möchte aber andererseits auch eine ägyptische Prinzessin zur Frau, ja er gibt sich sogar mit einer Ägypterin zufrieden, die nicht von königlichem Geblüt ist ${ }^{33}$. Die Absicht, die mit diesen Heiraten verbunden ist, wird unverblümt genannt: ana abbūuti u tābūti „wegen Brüderschaft und guter Freundschaft". Wie dem Gesamttenor der Briefe zu entnehmen ist, wurden hier aber nicht nur politische, sondern auch handfeste wirtschaftliche Vorteile gesucht. Vielleicht hat auch noch Burnaburiaš II. wegen der Übersendung einer Königstochter mit Amenopbis $I V$. in Verbindung gestanden ${ }^{34}$.

Dieser Burnaburiaš II. (ca. 1370-1343) ist auch sonst ein in der Heiratspolitik sehr reger Herrscher gewesen. Wahrscheinlich war es seine Tochter, mit Namen Mal-Nikkal, die als "Tawananna" die 3. Gemahlin Suppiluliumas I. von Hatti wurde ${ }^{35}$. Sie überlebte ihren Gatten, war noch unter Arnuwanda II. und Muršili II. Königinmutter und geriet wegen der in Hatti bedeutsamen Stellung der Königinmutter - mit der Gattin Muršilis in Streit. Es kam zum Prozeß, die alte Frau wurde „gedemütigt" und wahrscheinlich verbannt ${ }^{36}$. Es ist durchaus fraglich, ob ihr das gleiche Schicksal beschieden gewesen wäre, wenn sie nicht aus einer politischen Heirat heraus in Hatti zu einer Machtstellung gekom-

${ }^{28} K$. Riemschneider, ebd. S. 89 f. nach E. Forrer.

29 Telipinu-Erlaß $\$ 22$.

${ }^{30}$ Dazu K. Riemschneider, op. cit. (Anm. 27) S. 91 ff.

${ }^{31}$ Zusammenfassende und noch immer maßgebliche Edition durch J. A. Knudtzon, Die El-Amarna-Tafeln, in: Vorderasiatische Bibliothek Bd. 2 (1915) Texte 1-5. Die Briefe, die dem diplomatischen Archiv der Pharaonen Amenophis III. und Amenophis IV. Echnaton entstammen, sind dort nach ihrer Herkunft und chronologisch geordnet. Vgl. zuletzt C. Kühne, Die Chronologie der internationalen Korrespondenz von El-Amarna, in: Alter Orient u. Altes Testament 17 (1973).

${ }^{32}$ Knudtzon, ebd. Nr. 1, 11-14.

${ }^{33}$ Knudtzon, ebd. Nr. 4, 7 ff. Dieses Angebot ist recht beschämend für den Babylonier, der schreibt: „Erwachsene Töchter und schöne Frauen gibt es. Irgendeine schöne Frau, wie es dir gefällt, schicke! Wer wird denn (bier) sagen (können) ,Das ist keine Königstochter!'?" Kadaśman-Enlil mußte diese Bitte sogar noch einmal wiederholen. - Nach Knudtzon, ebd. Nr. 1,53 ff. hat der babylonische König auch noch an andere Fürsten Töchter zur Ehe vergeben, deren Namen wir aber nicht erfahren.

${ }^{34}$ Knudtzon, ebd. Nr. 11,5 ff.

35 Wir wissen lediglich, daß diese Gemahlin Suppiluliumas "Tochter des Königs von Babylon" war (E. Laroche, in: Ugaritica 3 [1956] S. 1 ff.), Chronologische Gründe sprechen für eine Abstammung von Burnaburiaš. 
men wäre, die der Fremden offenbar nicht neidlos eingeräumt wurde oder die sie mißbrauchte.

Burnaburiaš knüpfte aber auch verwandtschaftliche Beziehungen zu dem jetzt erstarkenden Assur an, vielleicht in der Hoffnung, auf diesem Wege von vornherein ein freundschaftliches Verhältnis der beiden Bruderstaaten zueinander in die Wege leiten zu können. Er heiratete die Muballițat-Šerua, eine Tochter Ašsur-uballițs, die ihm die zwei Prinzen Karakindaš und Kurigalzu șebru gebar ${ }^{37}$. Dies hatte zur Folge, daß nach dem Tode des Burnaburiaš, als der rechtmäßige Thronfolger Karakindaš durch den Usurpator Nazi$B u g a s ̌$ ermordet worden war, Aššur-uballit aktiv in die inneren Streitigkeiten Babyloniens eingriff, seinem Enkel Kurigalzu șebru zum Thron verhalf und damit natürlich auch einen wesentlichen Einfluß auf Babylonien bekam. Wir können vermuten, daß die Streitigkeiten, zu denen es nach dem Tode Ašsur-uballițs zwischen Enlil-nārārī und Kurigalzu II. kam, eben durch diese Einmischung gefördert wurden, ersehen daraus aber auch, daß das System der dynastischen Heiraten nur dann die gewünschte „Brüderlicbkeit und gute Freundschaft" zur Folge hatte, wenn die einzelnen Parteien daraus kein Recht auf Einmischung in die Angelegenheiten des nun verwandtschaftlich verbundenen Staates abzuleiten versuchten.

Verheerende Folgen hatte deshalb auch die ungewöhnlichste Heirat, die in dieser Zeit geplant wurde, schließlich aber nicht zustande kam: Diejenige eines hethitischen Prinzen mit der Witwe Tutenchamuns. Suppiluliuma berichtet in seinen "Taten", daß er bei der Belagerung von Karkemiš einen Brief der Witwe des Nibbururija erhielt des Inhalts: „Mein Mann ist gestorben. Einen Sobn habe ich nicht. Aber du, so sagt man, hast viele Söbne. Wenn du mir einen deiner Söhne geben würdest, würde er mein Gatte werden. Nie werde ich einen meiner Diener erwäblen und zu meinem Gatten machen." Es ist nicht verwunderlich, daß die Reaktion Suppiluliumas war: "So etwas ist mir in einem ganzen Leben noch nicht vorgekommen" 38 . Dennoch sendet er seinen Kammerherrn und bringt die Verhandlung in Gang. Die Angelegenheit scheint in Ordnung zu sein, denn nach mehreren Monaten wird der Prinz Zannanza geschickt, um Gatte der Anchesamun zu werden. Unterwegs aber wird er, wahrscheinlich auf Veranlassung des Harembeb, ermordet. Suppiluliuma führt daraufhin den Rachefeldzug gegen Agypten, der militärisch nicht zum Erfolg führen konnte, wegen der Verbreitung der Pest, die er zur Folge hatte, aber fast den ganzen Vorderen Orient entvölkerte ${ }^{39}$. Ungewöhnlich an dieser angestrebten Heirat ist fast alles: Die Frau lädt ein; der als Schwiegervater Erbetene ist Fürst der unmittelbar angrenzenden und in kurzer Zeit zu bedrohlicher Vormachtstellung gelangten Macht Hatti. Der Traum einer Großmacht vom Schwarzen Meer bis zum Roten Meer ist selbst in der Amarnazeit schwerlich geträumt worden, vielmehr zeugt dieser Vorschlag von erstaunlicher Naivität: Der König mit den vielen Söhnen ist der erwünschte Schwiegervater. Daß Suppiluliuma auf diesen Vorschlag einging, daß die Ägypter offenbar die hethitischen Diplomaten so gut zu täuschen verstanden, das ist das eigentlich Erstaunliche an diesem Vorgang. Er blieb Episode.

Die Folgezeit bringt harte Kämpfe zwischen Hatti und Ägypten, die erst nach der Qadešschlacht 1285 und dem Vertrag zwischen Ramses II. und Hattušili III. beendet

${ }^{36}$ H.-G. Güterbock, Siegel aus Boğazköy 1 (1940) S. 12 f.; E. Laroche (s. Anm. 35), S. 101-103.

${ }^{37}$ Quellen: Chronik P I $5 \mathrm{ff}$. und Synchronistische Geschichte I $8 \mathrm{ff}$. , vgl. dazu und zu den Folgerungen: W. Röllig, in: Heidelberger Studien zum Alten Orient (1967) S. 173 ff.

${ }^{38}$ "Deeds of Suppiluliuma" nach H.-G. Güterbock, in: Journ. of Cun. Stud. 10 (1956) S. $94 \mathrm{ff}$. III 7 - IV 15; 107 f. Fragm. 29-32.

39 Vgl. etwa W. Helck, Die Beziehungen Agyptens zu Vorderasien (2. Aufl. 1971) S. 183 f.; K. A. Kitchen, Suppiluliuma and the Amarna Pharaos (1962) S. 48. 
werden. Es ist deshalb nur logisch, daß erst zu diesem Zeitpunkt eine Tochter Hattušilis (mit der Königin Pudubepa) in den Harem Ramses II. geschickt wird, einige Zeit später eine zweite, um das gute Verhältnis der beiden Großmächte zueinander zu dokumentieren $^{40}$.

Auch sonst waren während des Neuen Reiches häufig fremdländische Mädchen im $\mathrm{Ha}$ rem der Pharaonen anzutreffen, vor allem Königstöcher aus Mitanni: Artatama I. gab eine Tochter an Thutmosis IV.41, Suttarna II. seine Tochter Gilu-Hepa an Pharao Amenophis III. in dessen 10. Jahr (1392) ${ }^{42}$. Dem gleichen Pharao wurde in seinem 36. Regierungsjahr auch noch die Tochter des Tušratta namens Dadu-Hepa zur Ehe gegeben ${ }^{43}$. Alle

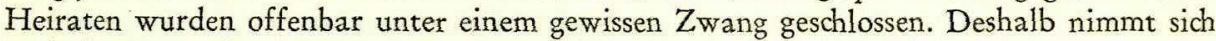
nach der Ermordung des Tušratta Suppiluliuma des Sohnes und Erben Kurtiwaza an, der zu ihm flieht - und zu seinem Schwiegersohn wird. Ramses II. hatte neben den hethitischen Prinzessinnen auch noch Königstöchter aus Babylon und Zulabi(?) in seinem Frauenhaus ${ }^{44}$.

Ägypten vergab Prinzessinnen offenbar nie ins Ausland. So hatte schon Amenophis III. an Kadašman-Enlil geschrieben: „Von alters ber wird eine Königstochter Agyptens an niemanden vergeben " 45 . Selbst Ugarit, durch seinen Handel schon lange mit Ägypten freundschaftlich verbunden, dürfte da keine Ausnahme gemacht haben. Zwar kennen wir die sog. "Hochzeitsvase" des Niqmadu II., die eine Hochzeit zwischen diesem Herrscher von Ugarit und einer Agypterin im 36. Jahr Amenophis III. (1366 v. Chr.) feiert; die Ansicht, daß es sich bei der Ägypterin um eine Prinzessin handelte, die etwa C. Schaeffer vertrat ${ }^{46}$, läßt sich aber durch den Schmudk der Kartusche widerlegen, der nicht dem einer ägyptischen Prinzessin entspricht ${ }^{47}$. Demnach wurde auch hierher nicht eine Königstochter, sondern wohl nur eine Haremsdame vergeben. Daß sich diese Politik offenbar später änderte, lehrt das AT, denn bekanntlich hat Salomo eine ägyptische Prinzessin zur Frau gehabt $^{48}$ - wenn wir hier der Uberlieferung trauen dürfen.

${ }^{40}$ In seinem 34. Regierungsjahr nahm Ramses II, nach dem Tode der Königin statt ihrer die hethitische Prinzessin zur Frau. In harten Auseinandersetzungen, die uns in der „Heiratskorresponden $z^{\text {" }}$ uberliefert sind, bekam sie auch das Recht, wie eine hethitische Königin eine eigene Korrespondenz zu unterhalten. Die Heirat wurde auch in einer Stele in Abusimbel festgehalten, s. dazu C. Kuentz, in: Annales du Service des Antiquités de l'Égypte 25 (1925) $181 \mathrm{ff}$; G. Lefèbore, ebd. 34 ff. Zur Heiratskorrespondenz s. vor allem E. Edel, in: Jahrb. f. Kleinasiat. Forschg. 2 (1953) S. 262 ff.; ders., in: Geschichte und A. T. - Festschrift A. Alt (1953) 50 ff. Die Vergabe der zweiten Tochter zusammen mit einer reichen Mitgift ist nur aus ägyptischen Quellen bekannt, s. W. Helck, op. cit. (Anm. 39) S. 221 f. mit Literatur.

41 Knudtzon, op. cit. (Anm. 31) Nr. 29, 16 ff.

42 Knudtzon, ebd. Nr. 29, 18-20; 17, 5 f. 26. Ein ägyptischer Text (W. Helck, Urkunden des ägyptischen Altertums 4/6 [1957] 1738, 14) erwähnt 317 Sklavinnen, die die Königstochter beim Einzug in den Harem begleiteten.

${ }^{43}$ Diese Nichte der Gilu-Hepa wurde gemäß der Korrespondenz (Knudtzon, ebd. Nr. 22.27.29) offenbar deshalb nach Ägypten vergeben, weil reiche Gegengaben erwartet wurden.

44 E. Edel, Geschichte, op. cit. (Anm. 40) S. $37 \mathrm{ff}$.

45 Knudtzon, op. cit. (Anm. 31) Nr. 4, Z. 6 f.

46 Publikation und Deutung der Vase durch Cl. F.-A. Schaeffer, in: Ugaritica 3 (1956) $164 \mathrm{ff}$. und $\mathrm{C} h$. Desroches-Noblecourt, ebd. S. $179 \mathrm{ff}$.

${ }^{47} \mathrm{H}$. Klengel, Geschichte Syriens 2 (1969) S. $344 \mathrm{f}$.

48 1. Reg. 3, 1; 9, 16. Der ägypt. Brautvater war vielleicht Psusennes II., s. A. Malamat, Journal of Near Eastern Studies 22 (1963) S. 9 ff. 
Das Neue Reich der Hethiter benutzte die Gelegenheit, durch Heiraten politisch Verbündete zu schaffen oder zu halten, Vasallen ans Reich zu binden und beaufsichtigen zu lassen, sehr viel freigebiger. Von den Verbindungen zu Babylon und Ägypten hörten wir schon. Suppiluliuma baute offenbar besonders auf Heiratspolitik, nicht immer mit Erfolg. So nimmt er den Mašbuiluwa von Mirâ als Flüchtling am Hofe auf und gibt ihm seine Tochter Murwatti zur Frau, ja Mursili II. setzt sogar seinen Thronanspruch durch ${ }^{49}$ trotzdem kommt es wohl zum Abfall des Vasallen. Als Kurtiwaza, Sohn des Tušratta von Mitanni, nach der Ermordung seines Vaters an den hethitischen Hof floh, wurde er ebenfalls mit einer Tochter Suppiluliumas verheiratet ${ }^{50}$, und sein Schwiegervater eroberte ihm das Reich zurück, das er dann - wenn auch nur kurz - selbständig regieren durfte. Eine Schwester des Hethiterkönigs wurde schließlich an Huqqanā von Azzi/Hajaša gegeben ${ }^{51}$, wobei gewisse Schwierigkeiten auftauchten: Die Regeln der geschlechtlichen Tabuierung von Blutsverwandten, die bei den Hethitern bestanden und streng eingehalten wurden, galten bei dem im armenischen Bergland wohnenden Volke offenbar nicht. Der Staatsvertrag enthält deshalb auch Regeln für den Umgang mit der zukünftigen Frau, mit der hethitischen Königsfamilie und über die Beschränkungen, die sich der Herrscher zukünftig bei der Wahl seiner Partnerinnen aufzulegen hatte. Illi-IR-inna, eine Tochter des Nachfolgers des Suppiluliuma, Muršili II., wurde an den Fürsten Mašturi vom ŠehaFluß vergeben ${ }^{52}$.

Besonders eng wurde der Pufferstaat Amurru in Nordsyrien, wohlbekannt durch die Aktivitäten des Abdi-aširta und Aziru während der frühen Amarnazeit, durch Heiraten an Hatti gebunden. Hattušili III. (1278-1250 v. Chr.) schloß mit Bentešina von Amurru nach dessen reuiger Rückkehr von ägyptischer Heerfolge einen Vasallenvertrag ab und gab ihm gleichzeitig die hethitische Prinzessin Gašsulijareija zur Frau, nahm aber auch eine der Töchter Bentešinas für seinen Sohn Nerikkaili entgegen ${ }^{53}$. Interessant ist nun, daß hier das Vasallenverhältnis recht schamlos ausgenutzt wird: Der Vertrag sieht ausdrücklich vor, daß die hethitische Prinzessin in Amurru Königin werden muß - obgleich dort schon seit Jahren die Gattin des Bentešina auf dem Thron saß. Für Sohn oder Enkel der neuen Königin ist der Anspruch auf den Thron festgelegt - Bedingungen, wie sie sonst nirgends überliefert sind. Sie wurden übrigens auch eingehalten, denn Šaušgamuwa, Sohn Bentešinas und der Hethiterin, besteigt den Thron und wird bei dieser Gelegenheit durch einen neuen Vasallenvertrag gebunden. Das Bündnis wurde auch noch durch die Heirat mit einer Schwester des Tudbalija IV., seines Vertragspartners, besiegelt ${ }^{54}$. Damit ist er in der etwas pikanten Situation, daß er eine Schwester oder Halbschwester seiner Mutter heiraten muß - ein Verhältnis, dem schon dadurch Rechnung getragen wird, daß der Vasallenvertrag keine Regelung der Nachfolge enthält, also auch keine Nachkommen erwartet wurden ${ }^{55}$. Hier werden aber auch die Grenzen dessen deutlich, was eine Heiratspolitik vermochte, die glaubte, sich über alle menschlichen Beziehungen hinwegsetzen zu können.

49 Nach der Einleitung des Vertrages Muršilis II. mit Kupanta-KAL von Mirâ und Kuwalija, s. J. Friedrich, in: Mittlg. der Vorderasiatisch-Aegypt. Gesellschaft 31/1 (1926) S. $106 \mathrm{ff}$.

${ }_{50}$ Vertrag Suppiluliuma-Kurtiwaza, s. E. Weidner, Politische Dokumente aus Kleinasien 1 (1923)

S. $18 \mathrm{ff}$.

${ }^{51}$ J. Friedrich, in: Mittlg. der Vorderasiatisch-Aegypt. Gesellschaft 34/1 (1930) S. $124 \mathrm{ff}$.

52 P. Meriggi, in: Wiener Zeitschr. für die Kunde des Morgenlandes 58 (1962) S. $70 \mathrm{ff}$.

${ }_{53}$ Vertrag Hattušili III.-Bentešina, s. E. Weidner, op. cit. (Anm. 50) S. $124 \mathrm{ff}$.

${ }^{54}$ Keilschrifturkunden aus Boghazköj 23 (1929) Nr. 1, vgl. 8 (1924) Nr. 82.

55 Vgl. hierzu H. Klengel, Geschichte Syriens 2, S. 320 f. 
Vielleicht war dies auch der Grund für das Scheitern einer weiteren politischen Ehe. Zwischen dem eben genannten Amurru und der reichen Hafenstadt Ugarit bestanden enge Verbindungen, denn beide Staaten lagen nahe beieinander. Niqmepa, vierter der durch die Archive bekannten Regenten Ugarits, war mit Abat-milku, vielleicht der Tochter des $D U-T e s u b$ von Amurru, verheiratet ${ }^{56}$. Sein Nachfolger Ammistamru II. heiratete dann eine Tochter des Bentešina von Amurru, die ihm auch den Thronfolger Utrišarruma gebar. Viele Jahre später, als in Amurru bereits Šaušgamuwa regierte, ließ sich Ammistamru von dieser Frau scheiden, weil sie ihrem Gemahl „Böses“ zugefügt hatte, wahrscheinlich auf politischem Gebiet ${ }^{57}$. Sie soll dann Ugarit mit dem Gut, das sie mit in die Ehe brachte, verlassen; der Kronprinz kann entscheiden, ob er ihr unter Verzicht auf den Thron folgen oder sich von seiner Mutter trennen will. In diese Affäre wird sogar Hatti eingeschaltet, das zwar vertraglich und familiär an Amurru gebunden ist, dennoch für Ammistamru entscheidet. Den Frieden zwischen Ugarit und Amurru muß Tudbalija auch in der Affäre der "Tochter der Großen Dame“, die aus Amurru stammte und am Hofe in Ugarit des Ehebruchs bezichtigt wurde, wiederherstellen ${ }^{58}$. Die Konsequenzen der engen familiären Verflechtung durch die konsequent angewandte Heiratspolitik sind hier deutlich ablesbar: Rechtsfälle, die im allgemeinen im privaten Bereich liegen und darin gesetzliche Regelung erfahren, weiten sich zu politischen Konflikten aus oder haben wenigstens eine Tendenz dazu. Politisch bindet aber eine solche Ehe, und zwar beide Partner; ihr Handlungsspielraum ist begrenzt.

Aus der Folgezeit sind nur noch wenige Nachrichten über dynastische Verbindungen erhalten; das politische Gesicht Vorderasiens hat sich ja auch entscheidend geändert. Zunächst stehen sich die feindlichen Brüder Babylon und Assur gegenüber, der König von Assur hat aber deutlich die stärkere Position. Um 1066 wird deshalb Adad-apla-iddina, „der Sobn eines Niemand“, in Babylon von Ašsur-bèl-kala von Assyrien auf den Thron gebracht. Als Dank dafür erhält er dessen Tochter zur Frau, und „das Volk von Assyrien und Babylonien vermischte sich (freundschaftlich) miteinander"59. Von einem Abhängigkeitsverhältnis des einen vom anderen erfahren wir nichts. Der Babylonier Nab̂́-šumaukin I. (904-888) lag - wenn wir der Synchronistischen Geschichte trauen dürfen - mit Adad-nīrārī II. längere Zeit in Fehde. Es kam dann aber auch hier zu einem Austausch von Königstöchtern, die gegenseitig zur Ehe gegeben wurden, woraufhin die Beziehungen offenbar friedlich wurden ${ }^{60}$. Aus neuassyrischer Zeit wissen wir davon, daß Sargon II.

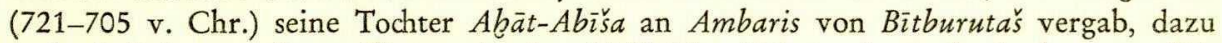
noch als Lehen an diesen Fürsten das Land Hilakku. 713 wurde Ambaris abgesetzt. Die Königstochter verwaltete aber offenbar zusammen mit einem rab bīti (Majordomus) die

${ }^{56}$ Bezeugung durch eine Liste mit Gerätschaften der Ahat-milku mit dem (dynastischen?) Siegel des DU-Tešub, s. J. Nougayrol, Palais Royale d'Ugarit 3 (1955) S. 182 ff.; ebd. 4 (1956) S. 120 ff.; C. F.-A. Schaeffer, Ugaritica 3 (1956) S. 28.

57 J. Nougayrol, Palais royal d'Ugarit 4 (1956) S. $126 \mathrm{ff}$; ders., Iraq 25 (1963) S. $115 \mathrm{ff}$.; $R$. Yaron, in: Orientalia 32 (1963) S. $22 \mathrm{ff}$; H. Klengel, Geschichte Syriens 2, S. 223, 322 f., $382 \mathrm{ff.}$

58 J. Nougayrol, ebd. 4, S. 129 ff.; H. Klengel, ebd. 2, S. 385 f.

59 Synchronistische Geschichte, Cuneiform Texts... in the British Museum 34, pl. 39 II $31 \mathrm{ff}$;; vgl. auch J. A. Brinkmann, A Political History of Post-Kassite Babylonia, Analecta Orientalia 43 (1968) S. $141 \mathrm{f}$.

${ }^{60}$ Cuneiform Texts... 34, pl. 40 III 17-19, s. J. A. Brinkmann (Anm. 59) S. 180 f. und 293 Anm. 1913. 
damalige Provinz Tabal weiter ${ }^{61}$. Wir können daraus ersehen, daß die Funktion, die eine Königstochter bei der Heirat mit einem abhängigen Fürsten übernahm, auch dann noch beibehalten werden konnte, wenn sie von ihrem Manne getrennt wurde, daß also die Stellung der Königstochter ungleich stärker war als die ihres Mannes. Uberliefert ist schließlich, daß Asarbaddon eine seiner Töchter dem Skythenhäuptling Bartatua geben wollte ${ }^{62}$, um an der Ostgrenze den Frieden zu sichern, den er auch beim Abschluß seiner Vasallenverträge für seine Nachfolge so dringend brauchte ${ }^{63}$.

Von den Chaldäerkönigen hat Nebukadnezar durch seine Heirat mit Amytis, der Tochter des Astyages von Medien, für die er angeblich die hängenden Gärten in Babylon schuf, legendären Ruhm gewonnen ${ }^{64}$. Wir können allerdings diese Königin ebensowenig wie die angebliche Nitokris, die eine ägyptische Prinzessin gewesen sein soll ${ }^{65}$, urkundlich nachweisen $^{66}$. Wie wichtig aber auch damals noch eine politische Hochzeit war, geht aus dem Bericht des Herodot über den Friedensschluß zwischen dem Mederkönig Astyages und Alyattes von Lydien hervor, der nach der berühmten Schlacht am Halys mit der Sonnenfinsternis vom 28. Mai 585 zustande kam. Die Vermittler beim Friedensschluß, der Kilikier Syennesis und der Babylonier Labynetos (Nabonid) bestanden darauf, daß Alyattes seine Tochter dem Astyages zur Frau gab, „weil ein Friede obne eine solche Verschwägerung wobl nicht von Dauer sein würde"67.

Wir könnten hier schließen, da die wesentlichen Aspekte der politischen Heirat im Alten Orient zur Sprache gekommen sind. Einen Komplex möchte ich aber nicht auslassen, der nicht immer ganz eindeutig ist: Die Heirat mit Frauen eines Volksstammes, um dessen Integration zu erreichen. Hier sind vielleicht zwei Namen zu nennen: Semiramis und Nāqia-Zakütu.

Uber Semiramis hat W. Eilers erst kürzlich eine vorzügliche Studie vorgelegt ${ }^{68}$. W. Schramm hat nochmals nachgewiesen, daß sie niemals selbständige assyrische Regentin war69. Trotzdem war ihr Einfluß und vor allem ihre Nachwirkung offenbar groß. W. Eilers hat angenommen, daß diese Königin aus dem Osten des Reiches, vielleicht sogar aus Urartu stammt, zur Befriedung dieses Reichsteiles von Šam̌̌si-Adad V. zur Ehe genommen wurde. Dem steht nun entgegen, daß alles nach dem Westen weist, wo die SemiramisLegende offenbar beheimatet ist. Nach Diodor ist Semiramis ja Tochter der Derketo, der Stadtgöttin von Askalon ${ }^{70}$, was sich jetzt sehr schön aus dem Ugaritischen erklären läßt. Dort wird die Göttin Anat als b'lt drkt und $b^{\circ} l t$ š $\mathrm{mm} \mathrm{rmm}$, „Herrin der Herrschergezealt"

${ }^{61}$ Vgl. R. F. Harper, Assyrian and Babylonian Letters (1892-1914) Nr. 197, S. 26 ff., und s. M. Streck, in: Vorderasiatische Bibliothek 7 (1916) S. CCXXV; E. Forrer, Die Provinzeinteilung des assyr. Reiches (1920) S. 73 f.; J. N. Postgate, in: Iraq 35 (1973) S. 31.

${ }^{62}$ E. G. Klauber, Politisch-religiöse Texte aus der Sargonidenzeit (1913) Nr. 16. - Bartatua ist der in Herodot, Historien I 103, genannte Vater des Madyas namens Protothyes.

${ }^{63}$ Vgl. D. J. Wiseman, in: Iraq 20 (1958) S. 9 f.

${ }^{64}$ Die Nachricht stammt aus Berossos und ist mehrfach (auch mit den Namenformen Amoubea, Tochter des Azdabak, vgl. W. Eilers, in: Semiramis [1971] 57101) überliefert, s. P. Schnabel, Berossos und die babylonisch-hellenistische Literatur (1923) S. 270 f.; E. Unger, Babylon (1931) S. 217.

${ }^{65}$ So nach $R$. P. Dougherty, Nabonidus and Belshazzar, Yale Oriental Series, Researches 15 (1929) S. $38 \mathrm{ff}$.

${ }^{66} \mathrm{Zu}$ Nitokris s. W. Röllig, Beiträge zur Alten Geschichte, Festschrift F. Altheim 1 (1969) S. $127-135$.

${ }^{67}$ Herodot, Historien 1, 74.

${ }^{68}$ W. Eilers, Semiramis, Ósterr. Akademie d. Wissenschaften, phil. hist. Kl., Sitzungsberichte 274/2 (1971).

${ }^{69}$ W. Schramm, in: Historia 21 (1972) S. 513-521.

70 Diodor 2, 4 ff., s. W. Eilers, op. cit. (Anm. 68) S. $12 \mathrm{ff}$. 
und "Herrin des Hohen Himmels" gepriesen ${ }^{71}$, letzteres übrigens auch noch eine Quartierbezeichnung in Sidon ${ }^{72}$.Semiramis ist demnach wohl eine Erscheinungsform der Anat, der Name Sammu-ramat wurde vielleicht volksetymologisch ins Assyrische übernommen. Damit bekommt aber die Heirat mit dieser Frau nicht nur einen politischen Aspekt, sondern auch einen religionspolitischen. Wenn Salmanassar III., wie wir jetzt wohl vermuten dürfen, auch ohne es in strengem Sinne beweisen zu können, die zukünftige Königin, die Frau für seinen Sohn, von einem seiner Westfeldzüge mitbrachte, so wünschte er eine Verschmelzung dieses Gebietes mit Assyrien. Sie gelang letztlich nicht, zu groß waren die Eigeninteressen der syrisch-phönizischen Küstenstädte. Auch religiöser Einfluß, wie er vielleicht beabsichtigt war, kam nicht zustande. Wohl aber waren Persönlichkeit und Religion der fremden Königin offenbar so überraschend und wirksam, daß sie die Phantasie des Volkes anregten und zu reicher Legendenbildung Anlaß gaben.

Nāqi $i^{i} \bar{a}$-Zakūtu, die tatkräftige Gattin Sanheribs, ist nach allgemeiner Auffassung nach ihrem ersten Namen eine Aramäerin gewesen ${ }^{73}$. Ihre gewichtige Rolle am Hofe und bei der Verwaltung Babyloniens in Lahiru sollte offenbar zum Ausdruck bringen, daß das aramäische Element im Volke gleichwertig war dem ursprünglich assyrischen, das zahlenmäßig sicher längst in der Minderzahl war. Falls wir so das Hervorgehobensein der Königin richtig interpretieren, so haben wir hier eine folgerichtige Entwicklung des Gedankens der politischen Heirat vor uns:

Zunächst geschaffen zur Festigung der Dynastienfolge, der Kontinuität des Herrscherhauses, wurde die politische Heirat rasch zum Instrument des Ausgleichs und der Versöhnung zwischen großen Staaten und Kleinfürstentümern. Koalitionen, die militärisch und wirtschaftlich von Nutzen sein konnten, wurden hier unter mehr oder minder freiwilliger $\mathrm{Zu}$ stimmung der Beteiligten in die Wege geleitet oder befestigt. Dies führte in der Amarnazeit zu einem dichten Netz von politischen und privaten Bindungen, das zum Gleichgewicht der Staaten untereinander nicht wenig beitrug, andererseits aber die Gefahr der Einmischung in die inneren Angelegenheiten immer in sich barg. Auch in der Folgezeit wurde die politische Heirat als Zeichen der Befriedung und gegenseitigen Respektierung verwendet, offenbar aber nicht mehr in dem Umfang, wie er für die Amarnazeit dokumentiert ist. Die Entwicklung zur Integration einzelner Volksteile durch Einbeziehung ihrer Repräsentanten in die königliche Familie läßt sich mit den Namen Sammuramat und $N \bar{a} q i^{i} \bar{a}$ wenigstens andeuten. Es bleibt die Aufgabe künftiger Forschung, die hier grob gezogenen Linien zu verfeinern, die toten Knochen mit Fleisch zu bekleiden, um sie leben zu lassen.

71 Ugaritica 5 (1968) S. 551: RS 24. 252, 7, vgl. auch Ch. Viralleaud, ebd. S. 555; H. Gese, in: $H$. Geese-M. Höfner-K. Rudolph, Religionen Altsyriens, Altarabiens und der Mandäer: Religionen der Menschheit 10/2 (1970) S. 157 f., 214.

72 H. Donner-W. Röllig, Kanaanäische und aramäische Inschriften (3. Aufl. 1971) Nr. 15, s. Bd. 2, 24.

${ }^{73}$ Zuletzt A. Parrot-J. Nougayrol, in: Syria 33 (1956) S. 147-160; M. Dietrich, Die Aramäer Südbabyloniens in der Sargonidenzeit, in: Alter Orient und Altes Testament 7 (1970) S. 26, 43 Anm. 1. 